\title{
РАЗРАБОТКА МОДУАЯ «ЗАНЯТОСТЬ АУДИТОРИЙ ИНФОРМАЦИОННОЙ СИСТЕМЫ ИНТЕРАКТИВНОГО РАСПИСАНИЯ ЗАНЯТИЙ И ЭКЗАМЕНОВ" ФГБОУ ВО ГГНТУ
}

\author{
(C) Ахмадов H.A., Саитов A.P. \\ ГГНТУ им. акад. М.Д. Миллионщчикова, г. Грозньй
}

Цель статьи: разработка модуля занятость аудиторий в вузе. Важным фактором, обуславливающий необходимость разработки информационной системы, является отсутствие на рынке программного продукта или комплекса, позволяющий использовать его в качестве научно-исследовательской деятельности. В ходе исследования проведены: анализ научно-исследовательской деятельности преподавателей и сотрудников Грозненского государственного нефтяного технического университета имени академика М.Д. Миллионщикова; анализ существующих систем автоматизированного место рабочего преподавателя; анализ программного обеспечения, необходимый для разработки информационной системы, а также сравнительный анализ антивирусных программ. В результате исследования разработан модуль "Занятость аудиторий» информационной системы интерактивного расписания занятий и экзаменов ФГБОУ ВО ГГНТУ которая предоставит возможность преподавателям и студентам учебного заведения упростить связанную с организацией и планированием контактной учебной работы, заполнением и актуализацией рабочих программ дисциплин, расписанием лекций, семинаров, лабораторных работ, экзаменов, зачетов и т.п. Такая информационная система существенно повысит оперативность и эффективностью учебного процесса в ВУзах.

Ключевые слова: информационная система, научно-исследовательская деятельность, занятость аудиторий, интерактивность, контроль.

В настоящее время во многих университетах нашей страны активно разрабатываются и внедряются различные информационно-программные комплексы, ориентированные на работу в глобальной сети Интернет (веб-приложения). Внешний вид 
и функциональность официальных веб-приложений учебного заведения влияют на его конкурентоспособность и во многом определяют его облик в глазах студентов, сотрудников, и абитуриентов [1].

Возможно, что из-за сложной организации или ограниченности выделяемых средств, не всегда удается поддерживать все веб-приложения (которых очень много) в соответствии с современным развитием веб-технологий и внедрять в них новый функционал. Так, например, веб-приложение «Интерактивное расписание занятий и экзаменов» Грозненского государственного нефтяного технического университета, мог бы содержать некоторые элементы «Интерактивной занятости аудиторий», и это оценили бы все: преподаватели, студенты и сотрудники вуза. Так как многие из них планируют рабочую неделю в соответствии со своим индивидуальным расписанием, в котором отмечают будущие мероприятия, теоретические и практические занятия и т.П.

В связи с этим разработка модуля «Занятость аудиторий» является актуальной.

Задача автоматизации управления образовательным процессом в вузе характеризуется [2]:

•большим объемом многообразной обновленной информации от различных структурных подразделений ВУЗа, таких как учебный отдел, деканы, кафедры, АСУ и информационная безопасность;

-сложность соотнесения и нахождения вариантов и ограничений составления расписаний, степень и качество которых полностью зависят от опыта и умения(мастерства) ответственного сотрудника учебно-методического управления;

-столкновение интересов между основными участниками образовательного процесса: студентами и преподавателями, кафедрами - как основными собственниками специализированного оборудования и помещений для учебных занятий, что приводит к ограничению на свободное их использование в расписании;

-трудности приспособления универсальных алгоритмов планирования к потребностям конкретного образовательного учреждения, особенно с учетом современных условий обучения в ВУ3-аx. 
Для эффективного и качественного решения задачи планирования и совершенствования деятельности любого учреждения, используется анализ, моделирование и оптимизация его бизнес-процессов.

Реинжиниринг бизнес-процесса составления расписания в Грозненском государственном нефтяном техническом университете позволит сократить сроки составления расписания; повысить качество расписания; уменьшить количество задействованных служб и структур.

А также, мы полагаем, что эта модель даст возможность:

•увидеть и, в последующем, скорректировать будущую систему до того, как она будет реализована физически;

•уменьшить затраты на создание системы, если в этом есть необходимость;

•достичь взаимопонимания между всеми участниками учебного процесса;

•позволит оценить работу по времени и результатам;

•улучшить качество создаваемой системы.

Для эффективного и качественного решения задачи планирования и совершенствования деятельности любого учреждения, используется анализ, моделирование и оптимизация его бизнес- процессов.

В качестве программного обеспечения для разработки такой системы выбрана среда SublimeText3 [3] при создании интерфейса, и среда разработки phpStorm для серверной части разработки, локальный веб-сервер Open Server, языки программирования: HTML, CSS, JavaScript и PHP, а также для построения базы данных - система управления базой данных MySQL, для управления которой необходимо веб-приложение phpMyAdmin.

Основным языком для создания структуры сайта является HTML. Большая часть современных интернет технологий основана на давно используемом, самом дискутируемом языке HTML. Он был разработан для выполнения разметки и оформления документов, размещаемых на веб-страницах. Свои первые черты язык начал обретать в 1986 году. Толчком стало принятие Международной организацией по стандартизации (ISO) ISO-8879-стандарта - Standard Generalized Markup Language или, в сокращенном варианте - SGML. K нему прилагалось описание, в котором говорилось о том, что SGML предназначен 
для структурной разметки текста. Примечательно, что описания внешнего вида документа не предполагалось.

Исходя из этого, можно сделать вывод о том, что SGML не являлся системой для разметки текста и не располагал какого-либо списка структурных элементов языка, используемых в определенных условиях. Язык подразумевал описание синтаксиса написания главных элементов разметки. Спустя некоторое время они получили, хорошо известное сегодня название - «теги».

Вполне очевидной была потребность в создании языка, который:

- Описывал какой элемент в каких случаях разумно применить

- Содержал перечень элементов, с помощью которых можно создать документ, читаемый разными программами

Несмотря на то, что язык SGML, как и его схожие приложения, не получил особого развития, он и не был окончательно забыт. В 1991 году Европейский институт физики частиц объявил о необходимости разработки механизма, позволяющего передавать гипертекстовую информацию через Глобальную сеть. Именно SGML лег в основу будущего языка-Hyper Text Markup Language (HTML). C помощью него задаются необходимые метаданные, которые содержат информацию о документе (сайте), а также необходимую информацию для поисковых машин. Также создается разметка сайта, где и как будет располагаться тот или иной элемент. Все необходимые файлы, содержащие коды других языков, подключаются с помощью этого языка.

SublimeText3 является распространенным текстовым редактором, со многими функциями, такими как быстрая навигация, командная палитра, одновременное редактирование, автосохранения, высокая степень настраиваемой, проверка синтаксиса, возможность поиска по мере набора. Он прост в использовании, имеет минималистичный интерфейс (минимум визуального шума) и кучу плагинов, расширяющих его функционал.

Создав «скелет» сайта, ему нужно придать красивый внешний вид. Для этого я буду использовать каскадные таблицы стилей CSS, которые будут находиться в подключаемом файле. CSS (Cascading Style Sheets, каскадные таблицы стилей) - язык описания внешнего вида документа, созданного с использованием 
языка разметки. Языком разметки может быть XML, SVG, XUL, но обычно в этой роли выступает HTML.

Цель: разделить логическую структуру документа (напримep, HTML-документа) и описание внешнего вида.

- разные виды одного документа (экран, принтер, голос)

- более «богатый» визуальный язык

- сам документ упрощается

- упрощается поддержка и разработка

Первое упоминание: 1994 год - Хокон Виум Ли (Håkon Wium Lie). Далее - затишье, к разработке подключается Берт Бос (Bert Bos).

1995 год - интерес со стороны W3C.

После 1995 года: развитие CSS как языка и создание стандарта. Это нужно, чтобы разработчики браузеров включали поддержку возможностей CSS в свои продукты.

С помощью этого языка определяется положение всех элементов на странице, а также их оформление. Существуют расширения, построенные на основе CSS это SCSS, Sass, LESS. [4] Так как все эти расширения перед отображением на сайте компилируются в файл CSS, то я сразу использовал каскадные таблицы стилей.

Чтобы придать интерактивность страницам, используется язык JavaScript. Этот язык работает на стороне браузера, то есть непосредственно с пользователем. На данный момент не существует аналогов, которые могут заменить язык JavaScript. Хотя большую часть функционала можно сделать с помощью CSS3, но этого еще недостаточно для полного замещения JavaScript. Существует одна очень большая и популярная библиотека jQuery, созданная на основе JavaScript. Я буду использовать ее в своем проекте, так как на ней написано очень большое количество плагинов.

Каждый сайт или веб-приложение состоит из двух частей. Это клиентская часть и серверная часть. Часть «клиент» - это то, что каждый пользователь видит на экране, когда заходит на определенную страницу. То есть в роли клиента выступает веб-браузер. Пользователь использует веб-браузер, чтобы обращаться к серверу для получения какой-либо информации. Другими словами, пользователь выполняет какое-либо действие в браузере, например, хочет открыть страницу сайта. Веб-браузер 
формирует запрос и отправляет его серверу. Сервер обрабатывает этот запрос и отправляет браузеру ту страницу, которую пользователь запросил. Веб-браузер отображает ее.

Есть много языков, которые существуют для разработки серверной части сайта. Это может быть авторизация и регистрация пользователя, поиск информации по сайту и так далее. Самый популярный язык веб-программирования серверной части - это скриптовый язык РНР. Поддерживается большинством хостинг-провайдеров. Также одним из преимуществ данного языка является простота кода и его компактность. Так как язык скриптовый, то код можно вставить в любую часть сайта, в код HTML. Но большинство разработчиков делают наоборот: используют PHP код, для отображения HTML. Это позволяет лучше контролировать содержимое страницы и ее функционал

Open Server - это портативный локальный веб-сервер, имеющий многофункциональную программу управления и большой выбор подключенных компонентов [6]. Это полноценный профессиональный инструмент, созданный для веб-разработчиков на основе их рекомендаций и пожеланий для разработки, отладки и тестирования веб-проектов, а также для предоставления веб-сервисов в локальных сетях.

MySQL представляет собой одну из самых распространенных сегодня систем управления базами данных [5] в сети Интернет. Основными ее качествами является надежность, высокая скорость и гибкость. Немаловажной характеристикой системы является ее бесплатность.

Для управления сервером MySQL, как говорилось ранее, необходимо веб-приложение phpMyAdmin. Он позволяет через браузер осуществлять администрирование сервера MySQL, запускать команды SQL и просматривать содержимое таблиц и баз данных. [7]

Техническое обеспечения системы рассматривается со стороны разработчика и пользователя.

Разработчику, для создания и обеспечения работоспособности информационной системы, понадобятся следующие составляющие:

- персональный компьютер с периферийными устройствами; 
- интернет;

- сервер.

К периферийным устройствам относится следующая аппаратура: клавиатура, мышь, сканер, принтер.

Подключение к сети интернет на момент стадии разработки может не потребоваться, однако после запуска и тестирования системы подключение к интернету необходимо для ее поддержки.

Сервер используется в разработке для хранения файлов, ответа на запросы пользователей и выдачи запрашиваемой информации, обработки и выполнения скриптов, работы с базой данных. Контроль над работой сервера возлагается на системного администратора.

Со стороны пользователя для работы с информационной системой, необходим только персональный компьютер с периферийными устройствами и интернет. В отличии от разработчика, пользователю сразу нужен доступ к интернету для пользования с системой.

Основные процессы, выполняемые в системе, являются:

Сценарий работы с приложением максимально прост. После того, как администратор получает свои данные для доступа к приложению, он авторизуется на сайте. После этого пользователь начинает настраивать все, что ему необходимо. Если это преподаватель и он просто для себя хочет вести электронный журнал своей группы, проводить тесты, общаться со студентами и использовать весь функционал приложения, то ему достаточно создать себе аккаунт преподавателя. После этого нужно авторизоваться под созданным аккаунтом и использовать функционал для преподавателя.

Если в системе будет участвовать и другие преподаватели, то им достаточно авторизоваться и пользоваться системой, для них уже все настраивает администратор. Они могут только составлять тесты, изменять свою личную информацию и добавлять публикации, а также общаться со студентами и другими преподавателями.

Для студентов функционал более прост, они могут только просматривать свое расписание и контроль успеваемости. Также они могут общаться с другими пользователями. На рисунке 1 изображена схема процессов, выполняемых в системе. 


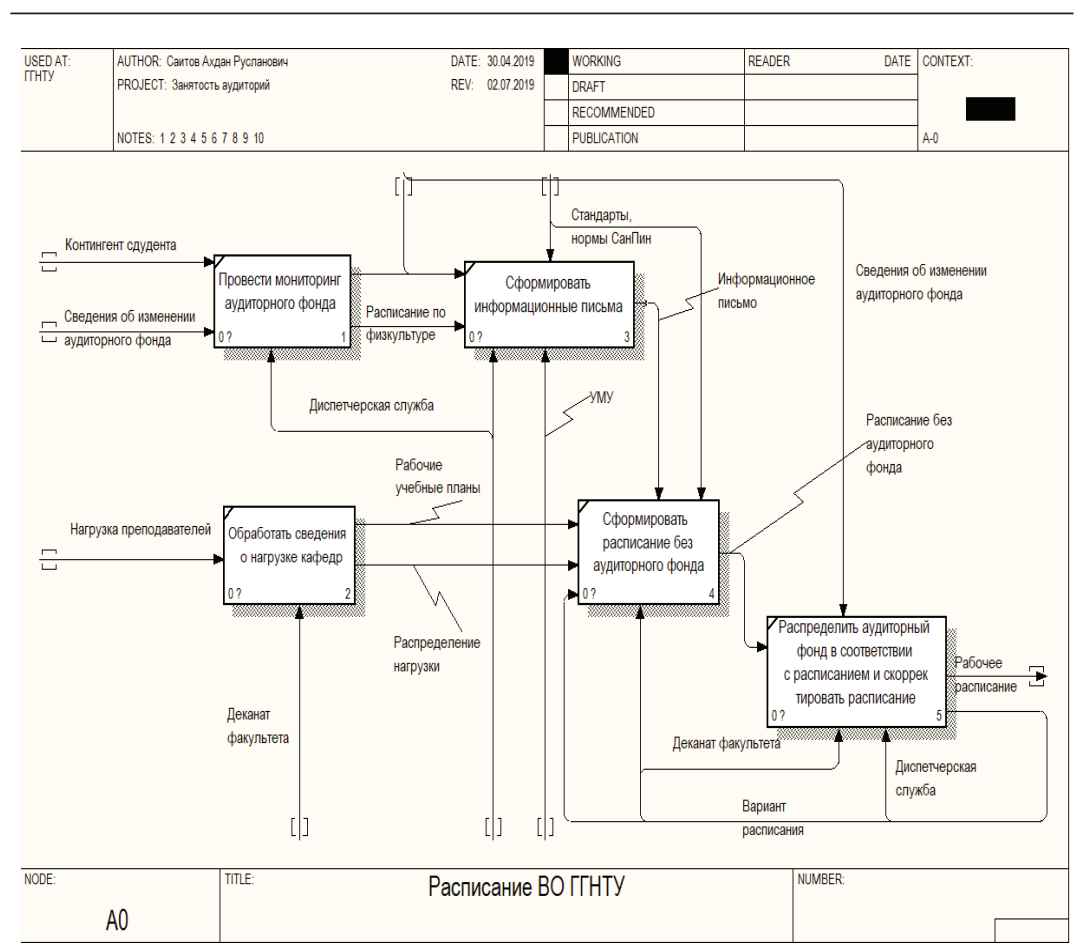

Рис. 1. Схема процессов, выполняемых в системе

В настоящее время разработка Web-сайтов становится все более популярным занятием, естественно, что в связи с этим, существует много способов и программ для создания веб-сайтов. Для разработки модуля «Занятость аудиторий» информационной системы интерактивного расписания занятий и экзаменов ФГБОУ ГГНТУ. Была использована платформа Sublime Text 3., так как считаем, что это очень современная, функциональная, продвинутая программа и кроссплатформный текстовой редактор. Работа началась с создания и разработки интерфейса с помощью HTML и CSS, что придает сайту динамику и привлекательный дизайн (рис 2)

А интерфейс, как мы знаем, предоставляет широкий набор инструментов для плодотворного взаимодействия человека и компьютерной системы. 


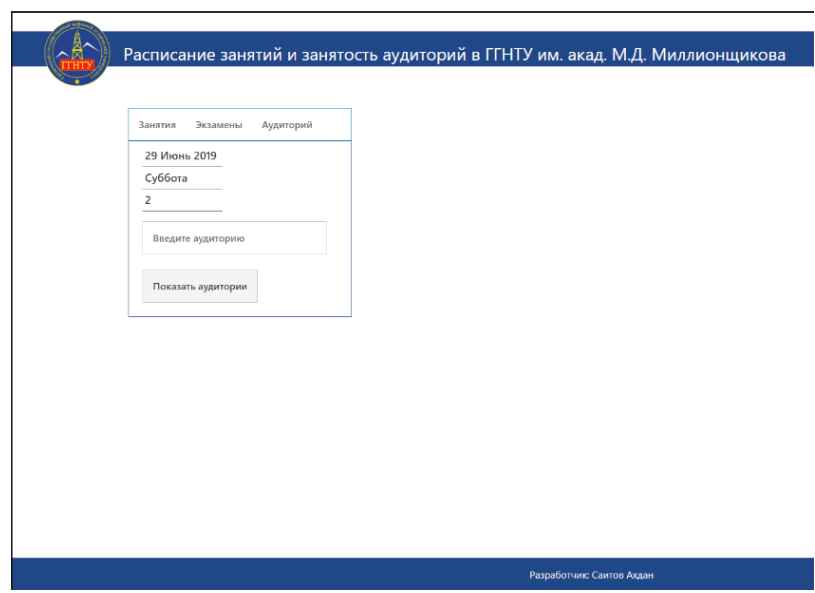

Рис. 2. Интерфейс

Для авторизации необходимо ввести логин и пароль. (рис 3) После входа на страницу заходим на раздел в правом верхнем углу «Панель администратора» (рис 4) «Панель администратора» этот раздел предназначен для админа.

\section{Авторизация}

Логин

Пароль

Вход

Рис. 3. Окно авторизацุии

Панель администратора Выход

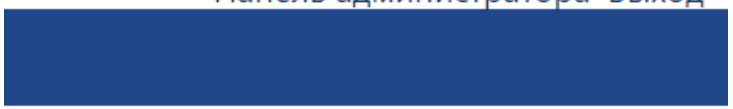

Рис. 4. Страница панель администратора 
На этой странице преподаватель может добавлять аудитории, группы, дисциплины, день недели и пары по своему усмотрению. После добавления информации, с помощью кнопки “Добавить занятия” (рис 5).
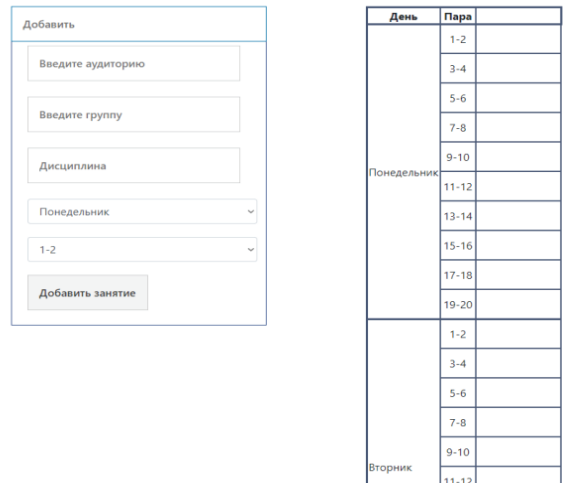

Рис. 5. Панель для добавлений занятий

После заполнении таблицы, добавляется таблица «Занятых аудиторий» (рис 6), после выполнения этого действия там мы имеем возможность увидеть - какие аудитории заняты, а какие в данное время свободны. Для удобства работы преподавателя в этой таблице автоматически, строки с занятыми аудиториями выделяются красный цветом.

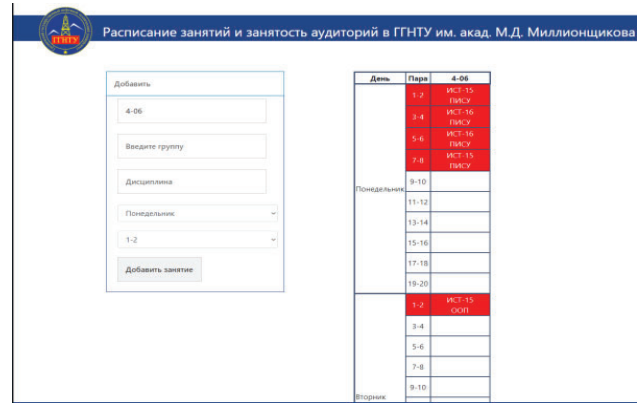

Рис. 6. Таблица занятых аудиторий 
Эта система также дает возможность студентам университета в любое время заходить на главную страницу и получить сведения о том в какой аудитории будут занятия или какие аудитории заняты/свободны. А еще такая программа позволит студентам университета в рабочее время использовать свободные от учебных занятий аудитории для самостоятельной или проектной, практической работы.

При разработке этой системы мы исходили из того, что она должна быть наглядной практично, функциональной и привычной не только после заполнения, но и во время работы

Мы использовали привычную для преподавателей и студентов таблицу, которую они часто видят в нашей вузовской системе.

На сегодняшний день, одной из обязательных характеристик любой информационной системы является средства обеспечения информационной безопасности, т.к. высокая степень автоматизации порождает риск снижения безопасности информации.

Для обеспечения мероприятий по обеспечению безопасности необходимо задействовать разные средства защиты. Они представляют собой комплекс, состоящий из совокупности различных устройств, технических систем и приборов, которые могут быть использованы для решения различных задач по предупреждению утечки информации, общей защите и обеспечения безопасности защищаемой информации.

Средства по обеспечению защиты информации, в частности прекращения умышленных действий, завсимости от способа реализации, делят на следующие группы:

- аппаратные технические средства (к ним относятся устройства различного типа, которые аппаратными средствами решают задачи по защите информации);

- программные средства (программные продукты для идентификации, шифрования информации, контроля доступа, удаления остаточной информации, тестового контроля системы защиты и др.).

Для обеспечения информационной безопасности разработанной системы были выбраны следующие средства защиты:

- Антивирусная защита. Для обеспечения безопасности си- 
стемы нам необходимо установить на сервер, в котором будет храниться данная система, подходящий антивирус, который предотвратил бы непредвиденные угрозы.

В настоящее время, наиболее надежными и эффективными средствами защиты программы, являются:

Антивирусные программы от компании ESET NOD32 (рис.7)

Компания ESET предлагает решения в сфере информационной безопасности, как для защиты ПК домашних пользователей, так и для серверов и рабочих станций компаний любого масштаба.

- Авторизация и аутентификация пользователей. При данной мере пользователь должен использовать процедуры входа в систему, как средство идентификации в начале работы. При таком подходе для определения личности любого пользователя нужно задавать уникальные логины и пароли, не являющиеся комбинированными данными личных данных пользователя.

В данной информационной системе, как говорилось ранее, разработана и внедрена процедура авторизации, которая определяет, кто из пользователей должен иметь доступ к той или иной информации и возможностям.

Использование таких средств защиты позволило свести к минимуму риск информационной безопасности.

Подводя итоги, отметим, что внедрение разработанной информационной системы позволит:

- повысить эффективность научно-исследовательской деятельности ВУЗа;

- предоставить возможность сотрудникам структурных подразделений вуза вести учет результатов своей научной деятельности;

- существенно улучшить процессы учета и контроля научно-исследовательской деятельности подразделений;

- сократить время подготовки и формирования отчетов различных видов;

- предоставить руководителям отдельных структурных подразделений и организации в целом средства проведения анализа научно-исследовательской деятельности каждого из сотрудников, отдельных подразделений и всей организации. 


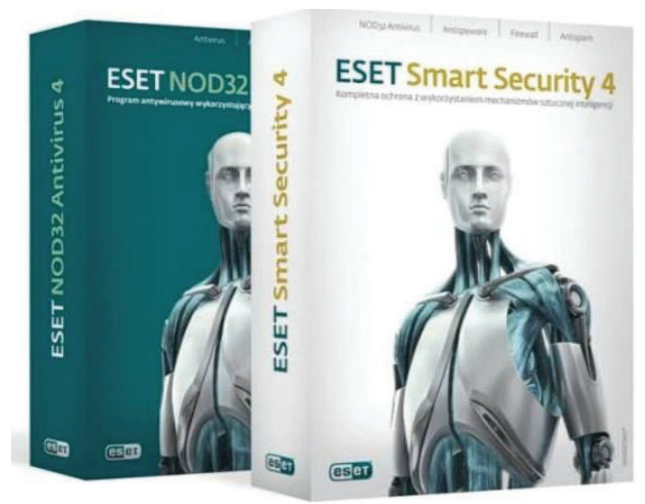

Рис. 7. ESET NOD 32 Antivirus

\section{Мнтература}

1. Якунин Ю.Ю. Технологии разработки программного обеспечения. Версия 1.0 лаб. практикум [Электронный ресурс]: / Режим доступа: http://files.lib.sfu-kras.ru/ebibl/umkd/183/u_lab. pdf. (дата обращения: 10.10.2020).

2. Положение о научно-исследовательской работе профессорско-преподавательского состава, утвержденное ректором ГГНТУ 14.10.2020 // Официальный сайт ГГНТУ. URL: http:// gstou.ru/files/localnie_akti/pologeniya/pologenie_o_nir_pps.pdf (дата обращения: 11.10.2020).

3. Описание Sublime Text 3 [Электронный ресурс] / Режим доступа: https://www.sublimetext.com/buy?v=3.0. (дата обращения: 14.10.2020).

4. Ефромеев Н.M. Основы web-программирования: учебное пособие/ Ефромеев Н.М., Ефромеева Е.В. - Электрон. текстовые данные. Саратов: Вузовское образование, 2019. 128 с. URL: http://www.iprbookshop.ru/86300.html (дата обращения: 15.10.2020).

5. Рыбин С.И. Способы связей в SQL: учебник / С.И. Рыбин. - СПб.: СпецЛит, 2014. - 312 c. URL: https://books.ifmo.ru/file/ pdf/1925.pdf (дата обращения: 15.10.2020).

6. Open Server - профессиональный инструмент веб-разработчика под Windows [Электронный ресурс] / Режим доступа: https://habr.com/ru/post/137388/. (Дата обращения: 15.10.2020).

7. Обзор антивирусных программ. [Электронный ресурс] / режим доступа: http://compress.ru/article.aspx?id.(дата обращения: 16.10 .2020$)$. 